\title{
SIWF-Award - für besonderes Engagement in der Weiterbildung
}

\author{
Werner Bauera, Raphael Stolzb ${ }^{b}$, adja Jennic \\ ${ }^{a}$ Dr. med., Präsident des Schweizerischen Instituts für ärztliche Weiter- und Fortbildung SIWF

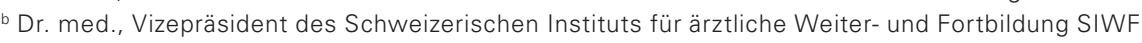 \\ ${ }^{c}$ M. Sc., Wissenschaftliche Mitarbeiterin SIWF/FMH
}

Wir freuen uns, zum dritten Mal die Ausschreibung für den SIWF-Award veröffentlichen zu können. Die zahlreichen und detaillierten Rückmeldungen haben uns den Wert dieser Auszeichnung gezeigt. Bereits nach dieser kurzen Zeit lässt sich die Verleihung nicht mehr aus der Agenda des SIWF wegdenken.

Entscheidende Grundlage für eine optimale Vermittlung von Können und Wissen an die Weiterzubildenden ist das Engagement der Kaderärztinnen und ärzte. Diese Aufgabe lässt sich nur beschränkt durch Pflichtenhefte definieren, ebenso wichtig sind persönliches Engagement und Begeisterung. Die Belastungen in der medizinischen Arbeitswelt sind vielfältig und die zeitlichen und finanziellen Ressourcen werden knapper, umso mehr sollten besonders aktive und motivierte Weiterbildnerinnen und -bildner auch eine Anerkennung erhalten. Deshalb bietet das SIWF den Assistenzärztinnen und -ärzten die Möglichkeit, das ausserordentliche Engagement von Weiterbildungsverantwortlichen ausdrücklich anzuerkennen, ohne aber eine Rangliste erstellen zu wollen.

Hat sich ein früherer Weiterbildner exemplarisch für Ihre Weiterbildung eingesetzt? Haben Sie dank der hohen didaktischen Kompetenz einer Weiterbildnerin speziell erfreuliche Fortschritte in Bezug auf Kenntnisse und Fähigkeiten machen können? Dann nomi-

\section{Jetzt Weiterbildungsverantwortliche nominieren!}

Der SIWF-Award gibt die Möglichkeit, besonders engagierten und kompetenten ärztlichen Weiterbildungsverantwortlichen eine Anerkennung zu übermitteln. Hat ein ehemaliger Weiterbildner oder eine ehemalige Weiterbildnerin bei Ihnen einen bleibenden Eindruck hinterlassen? Dann nominieren Sie ihn oder sie für den SIWF-Award für besonderes Engagement in der Weiterbildung!

Senden Sie Ihr Nominationsschreiben bitte in elektronischer Form an: siwf[at]fmh.ch mit dem Vermerk "SIWF-Award für besonderes Engagement in der Weiterbildung".

Einsendeschluss: 7. August 2016

Informationen finden Sie auch auf www.siwf.ch. Bei Fragen wenden Sie sich bitte an uns unter der E-Mail-Adresse siwf[at] fmh.ch oder der Telefonnummer 0313591111. nieren Sie diese engagierten Weiterbildungsverantwortlichen für den SIWF-Award für besonderes Engagement in der Weiterbildung!

\section{Nominierung durch Assistenzärztinnen und -ärzte}

Für den SIWF-Award können Personen nominiert werden, welche zurzeit in der ärztlichen Weiterbildung aktiv tätig sind. Im Fokus stehen Kaderärztinnen und

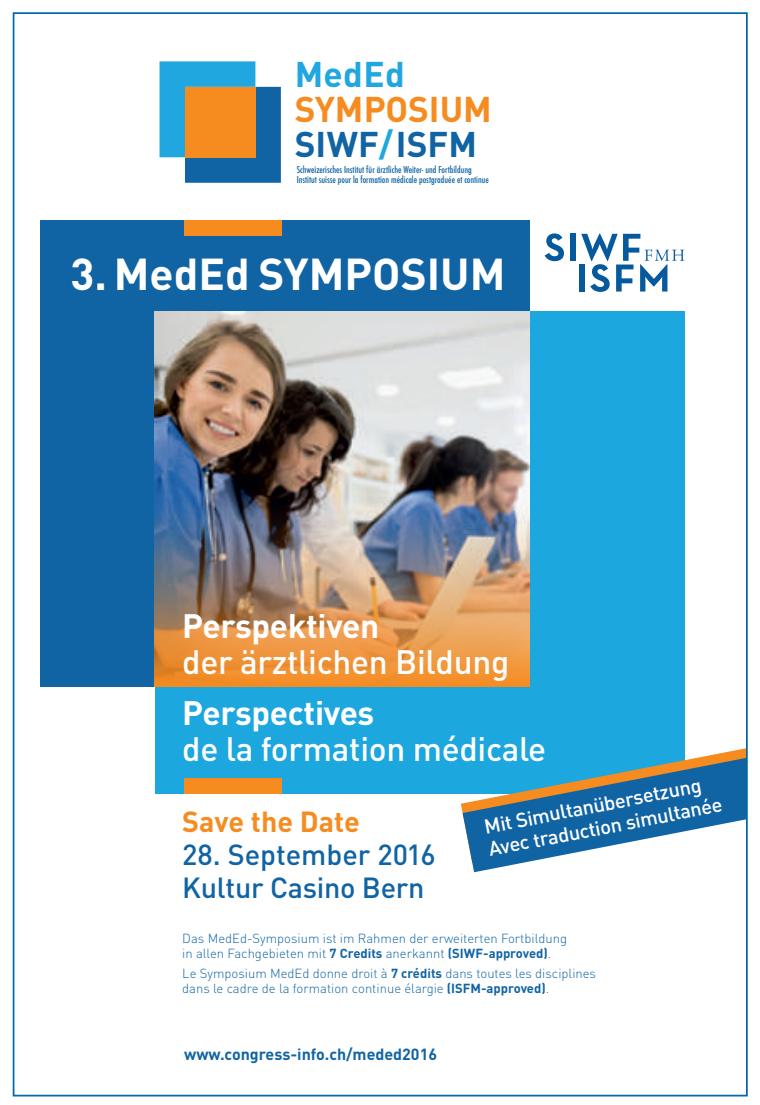


Korrespondenz:

Schweizerisches Institut

für ärztliche Weiter- und

Fortbildung SIWF

FMH

Elfenstrasse 18

Postfach 300

CH-3000 Bern 15

Tel. 0313591111

siwf[at]fmh.ch -ärzte, welche sich persönlich in der Weiterbildung von angehenden Fachärzten und -ärztinnen intensiv engagieren und von diesen in Bezug auf die Weitergabe von Kenntnissen und Fertigkeiten als besonders kompetent erlebt werden. Nominationsberechtigt sind deshalb Ärztinnen und Ärzte, die sich zurzeit in der Weiterbildung zu einem Facharzttitel befinden oder vor weniger als einem Jahr den Facharzttitel erworben haben. Eine Nomination ist dann gültig, wenn sie durch zwei Personen gemeinsam erfolgt. Sie soll die persönliche Wertschätzung für die wahrgenommene Weiterbildungsqualität und für das Engagement der Weiterbildungsverantwortlichen ausdrücken. Damit aufgrund des Nominationsprozesses keine Vorteile oder Konflikte am Arbeitsplatz entstehen können, dürfen nur Weiterbildungsverantwortliche nominiert werden, bei welchen die Nominierenden aktuell nicht mehr angestellt sind. Die Namen der nominierenden Personen werden nicht veröffentlicht und den Nominierten auch nicht mitgeteilt. Es wird keine «Rangliste» erstellt.

\section{Jetzt Nomination einreichen!}

Um jemanden zu nominieren, können Sie das vorgesehene Formular unter folgendem Link herunterladen: http://www.fmh.ch/bildung-siwf/weiterbildung/ allgemein/siwf-award.html

Das ausgefüllte Gesuchsformular senden Sie bitte in elektronischer Form an: siwf[at]fmh.ch

Das Nominationsschreiben können Sie bis am 7. August 2016 einreichen. Die SIWF-Geschäftsleitung überprüft, ob die Nominierung formell korrekt ist, und entscheidet abschliessend über die Gültigkeit der einzelnen Nominationen.

Alle korrekt Nominierten erhalten als Würdigung Ihres Engagements in der Weiterbildung eine Anerkennungsurkunde und ein Präsent. Sie werden (nach Rückfrage) auf der SIWF-Webseite (www.siwf.ch) aufgeführt und am MedEd-Symposium vom 28. September 2016 namentlich genannt. 